\title{
Prototype Digital Signage System for Learning Material Delivery Focusing on Student Friendship
}

\author{
https://doi.org/10.3991/ijet.v15i22.14171
}

\author{
Hiroyuki Mitsuhara ( $\left.{ }^{\bowtie}\right)$, Masami Shishibori \\ Tokushima University, Tokushima, Japan \\ mituhara@is.tokushima_u.ac.jp
}

\begin{abstract}
A digital signage system to deliver learning materials on a university campus is described. The proposed system identifies student viewers using a high-powered radio-frequency identification reader and delivers learning materials that are suitable for multiple students based on existing and potential friendships, which can be estimated from the learning material viewing data. The delivery of learning material can reinforce existing student friendships and identify potential friendships (i.e., unite unacquainted students who can potentially form a learning community). A preliminary experiment revealed that the existingfriendship estimation is currently inaccurate and unsuitable.
\end{abstract}

Keywords - Digital signage, learning material delivery, student friendships, university campus

\section{Introduction}

People can receive digital information in various ways. For example, they can receive web content, instant messages, and other digital information through smartphones anywhere at any time. People who do not use smartphones can also receive digital information via digital signage (DS). DS is an information delivery medium that uses computer displays in public spaces to target multiple unspecified people. Various DS systems that focus on networking, interactivity, and context-awareness as well as delivering digital information have been proposed [1]. For example, Communiplay [2] connects public displays installed in remote locations and enables viewers in other locations to interact through delivered digital content. A field study of Communiplay revealed that the interactivity attracted passersby by inducing visual effects. Ice Window [3] attempts to promote interaction between viewers in two locations connected via a real-time video by a gesture-based visual effect that represents melting window ice. By detecting a viewer's gaze focus, GazeHorizon [4] enables a viewer to select digital content from a scrollable menu and look at the content in the center of a public display. UBI Hotspot [5] delivers user-adapted digital content using Near Field Communication (NFC), a radio-frequency identification (RFID) reader, and two cameras. Field studies of UBI Hotspot in real-world environments showed that, for more effective deployment, interactive DS systems should focus on curiosity and novelty, location, social context, and weather. Recently, DS systems (or pervasive screens) have included smart 
devices (e.g., smartphones) to make information delivery more interactive and reachable [6]. For example, Hossain et al. [7] realized binding a public display and viewers' smartphones using Internet of Things technologies and invisible image sensor communication. Kim et al. [8] realized another binding method by obtaining a unique content ID from the content brightness changing pattern.

DS systems are frequently employed at educational facilities. For example, Brignull et al. [9] developed an interactive DS system that can be used to share and exchange multimedia data on a large public display. They reported that the system revitalized student social interactions through practical use in a high school. Other interactive DS systems have adopted touchless gestural interactivity. Source et al. [10] developed an interactive DS system that displays a visible avatar together with several kinds of information (e.g., news and lecture timetable) to attract university students to the display and promote understanding of the system interactivity. Focusing on embodied cognition, ELASTIC ${ }^{3} \mathrm{~S}[11]$ enables students to simulate and learn cross-cutting science concepts (e.g., earthquake mechanisms and effects) through their gestures.

The first author launched an educational DS project called "Niche-learning" and developed a DS system called the Niche-learning System (NLS) [12]. The NLS uses DS as a learning material delivery medium and attempts to encourage university students to learn informally during the break between lectures. The NLS delivers learning materials (e.g., slideshows and videos) using computer displays installed in public spaces (e.g., lounges and entrance halls) on university campuses. To attract student attention, the NLS superimposes the students onto a quiz slideshow and allows them to answer the quiz using body movements [13]. In addition, based on student viewing behaviors, the NLS can change the delivered learning material to different material that a single student can view from beginning to the end [14].

However, we believe that the NLS should have functionality for multiple students. In other words, the system should deliver learning material that is suitable for multiple students simultaneously. Related to this objective, we have considered delivering learning material that reinforces student friendships as a learning community and unites unacquainted students who can potentially form a learning community.

The remainder of this paper is organized as follows. Section 2 outlines the Nichelearning project and the NLS. Section 3 discusses the delivery of learning materials based on student friendship. Section 4 reports a preliminary experiment about student friendship estimation. Conclusions and suggestions for future work are presented in Section 5 .

\section{$2 \quad$ Niche-Learning}

\subsection{Overview}

Niche-learning is a phrase that is derived from the word "niche," which represents the short break time between lectures, and "e-learning." Although the break time between lectures is short, it can be considered an informal learning opportunity. Students often spend their break time with friends in a lounge area. Thus, if a DS system is 
installed in the lounge area or in lecture hall entranceways, they can learn from material delivered using DS if their circumstances permit such activities.

\subsection{System}

The NLS delivers learning materials according to a schedule set by an administrator. At a specified delivery time, the NLS presents the scheduled learning material using a video player or a slideshow viewer. Several NLSs were installed in lounge areas and entrance halls at Tokushima University. The installed systems have delivered learning materials (e.g., English conversation material) for four years. Figure 1 shows NLSs installed in a lounge area and lecture hall entrance. In Japan, typically, the break time between classes or lectures is less than 15 minutes, with the exception of the lunch break. Therefore, the learning materials presented by the NLS are less than 15 minutes in duration ( 5 minutes in most cases).

However, we found that many students did not view the learning materials because they did not have sufficient time during the break between lectures. Hardy et al. [15] classified the attention level of passersby to a public display as ignore, glance, and watch. According to this classification, it can be said that many students' attention level was ignore or glance. In addition, the following situations were also observed.

- Even with sufficient time, uninterested students do not view the learning material.

- Interested students view the learning material for a short period.

- If interested students begin viewing in the middle of the learning materials, they do not continue watching.

Although increasing the length of the break time (i.e., the frequency of learning material delivery) would likely improve these unexpected situations, this is often difficult or impractical due to university rules or traditions. Therefore, to address these issues, we have extended the NLS.
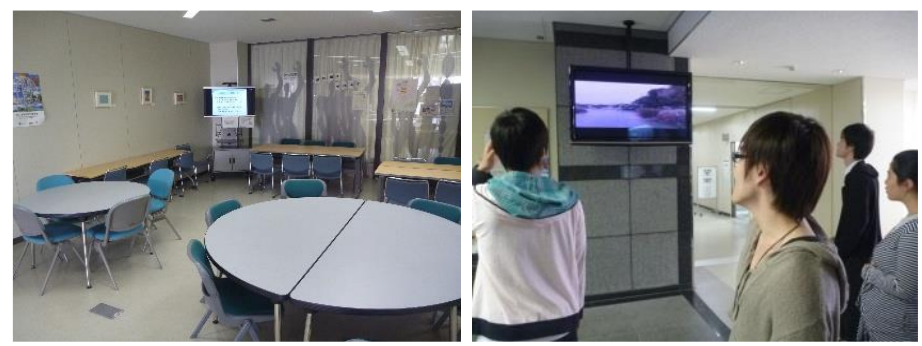

Fig. 1. Installed NLSs (left: lounge area, right: lecture hall entranceway)

\subsection{Extended system}

To extend the NLS, we have defined ideal learning using the NLS as follows: "A student views learning materials from the beginning to the end discontinuously." This 
definition embodies a learning continuum (LC) concept [14]. We have defined the following LCs.

- -LC1 (Single set of learning material): After partially viewing a learning material, the student can restart viewing the learning material from the beginning.

- LC2 (Multiple sets of learning material): After viewing a learning material from beginning to end, the student views other learning material from the available material. In other words, the student does not view the same learning material in succession.

Note that LC1 is a prerequisite for LC2. Therefore, it is important that the NLS allows students to view the learning material from the beginning. On the basis of LC1 and LC2, ideal learning means that a student views the materials completely from one learning material.

Figure 2 illustrates a case in which the LCs are satisfied. In this figure, a student (S1) views two sets of learning material (LM1 and LM2) using DS1 and DS2. Using DS1, S1 begins viewing approximately halfway through LM1 (300 s long), i.e., at $120 \mathrm{~s}$, and stops viewing after $120 \mathrm{~s}$. Using DS2, S1 begins viewing LM1 from the beginning and stops viewing after $180 \mathrm{~s}$. Using DS1, S1 continues viewing LM1 from the previous viewing stop point and views the content to the end. Thus, LC1 is satisfied for S1 and LM1. Using DS2, S1 views LM2 from the beginning. Thus, LC2 is satisfied for S1, LM1 and LM2 (LM1 to LM2). The NLS aims to facilitate the illustrated situations.

To satisfy the LCs, the NLS must be able to identify students. In addition, the system should be installed in many public spaces. As shown in Fig. 3, the NLS employs centralized client-server architecture for data exchange. Note that there are multiple client systems on campus. Each client system has a large display and communicates with the server system, which stores the learning materials, via the Internet. To satisfy the LCs, the server system functions as a data server.

- Client System: For student identification, each client system has a high-powered RFID reader. The area covered by the RFID reader is referred to as the viewable area. The viewable area forms an irregular fan with a radius of approximately $5 \mathrm{~m}$ in front of the display. Note that students must carry passive RFID tags for the system to function. The client system receives the IDs of students inside the viewable area from the RFID reader and sends viewing data, such as student ID (SID) and learning material ID (LMID), to the server system.

- Server System: The server system, which has a software interface between the client system and the database of student data, viewing data, and learning material data, selects the learning material to be delivered to the student identified by the client system. 


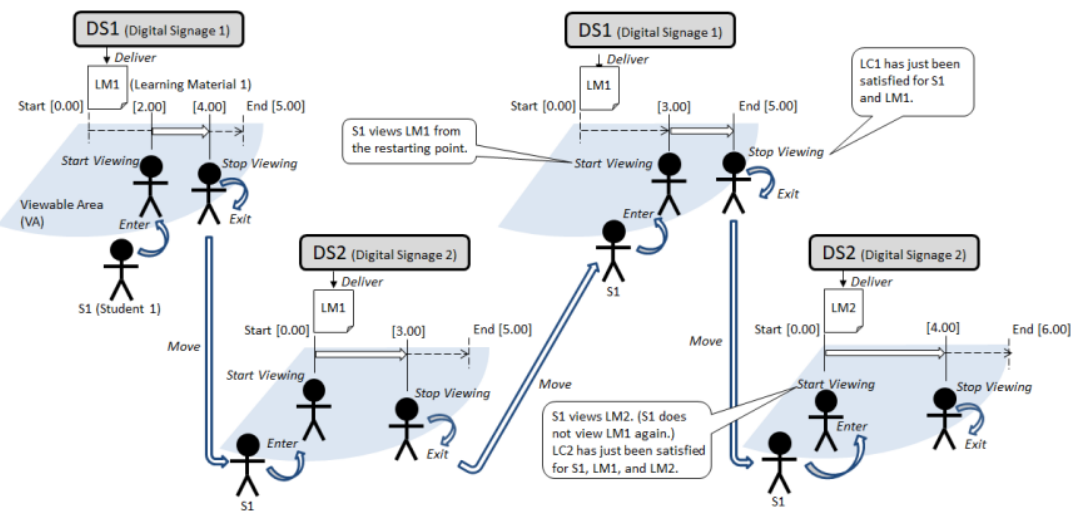

Fig. 2. Satisfying LC1 and LC2

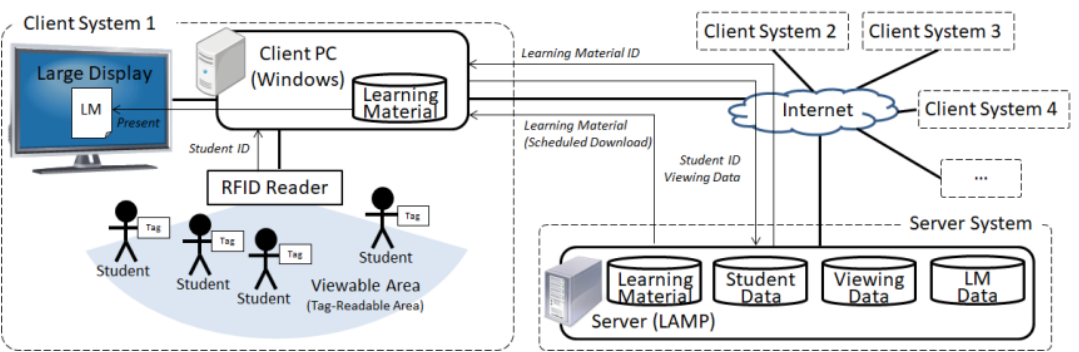

Fig. 3. Architecture of the extended NLS

\section{Learning Material Delivery Focusing on Student Friendship}

The LCs only considered a single student who entered the viewable area earlier than other students in each break time. Therefore, the NLS has been further extended so that the LCs can be applied to multiple students.

\subsection{Fundamental idea}

To satisfy the LCs for multiple students in the NLS, it is important to revise the LCs in consideration of the factors that motivate students to view learning materials in their entirety. Thus, we have focused on friendship as one such factor because students often spend break time with their friends. If one friend stops to view learning material, it is expected that all friends will also participate. Viewing the same learning material with friends simultaneously can prompt discussion regarding the learning material. In addition, we believe that friendships formed through face-to-face contact in the real world can motivate students to learn continually and collaboratively.

Here, we define the following new LCs (Fig. 4). 
- LC3 (Reinforcing existing friendship): When a student is with friends, they view learning material from the beginning to the end simultaneously. Then, the existing friendship is reinforced as a learning community. Consequently, they become accustomed to learning together more frequently, even in other situations.

- LC4 (Identifying potential friendship): When a student is with students with whom they are unacquainted, they view learning material from the beginning to the end simultaneously. In this situation, the students become aware of having common interests and are united through viewing the learning material. Then, these potential friendships become existing friendships and are identified as a learning community. Consequently, these students can learn together in other situations.

Based on the above fundamental ideas, we further extended the NLS to satisfy LC3 and LC4.

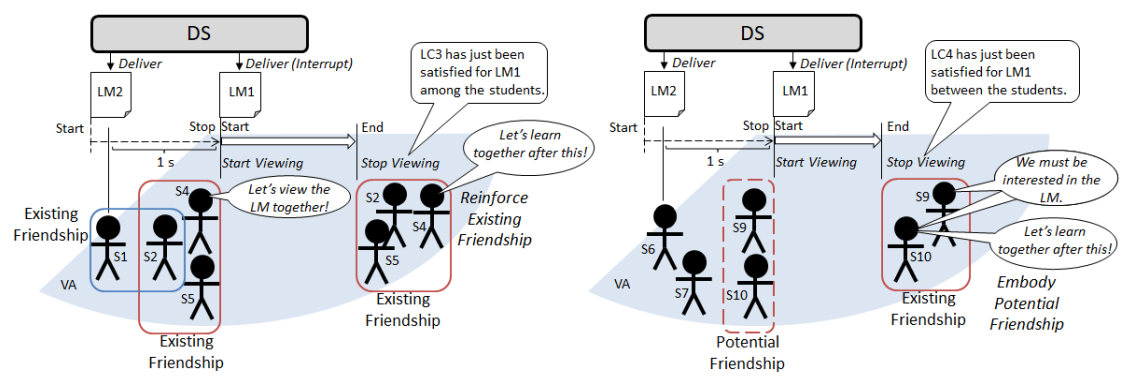

Fig. 4. Satisfying LC3 and LC4

\subsection{Student model and friendship estimation}

To satisfy LC3 and LC4, first, the extended NLS must build a student model that represents student friendships. The student model can be determined by the viewing data collected by the RFID reader. Example viewing data are listed in Table 1. In Table 1 , each represents student interest for the given learning material. If a student is interested in specific learning material, they will stop to view it. For simplification, we assume that, if a student is inside the viewable area for $1 \mathrm{~s}$ or more, they are interested in the learning material being delivered at that time. Under this assumption, the student is considered interested in the learning material even if they do not actually view the material. Presumably, the longer a student views the learning material, the more interested they are in the material.

Students with common interests have already been friends or can become friends in the near future. Thus, two types of friendship between students, i.e., existing and potential friendships, are estimated from the viewing data. Figure 5 shows an example of friendship estimation that is associated with Table 1. In Figure 5, the same learning material is delivered at the same time but in different places (DSID = 1 and 2). The student model represents the existing and potential friendships as a symmetric matrix. Table 2 shows an example of the friendship matrix that is associated with Table1. The above assumption does not exclude contingency, i.e., the possibility to inaccurately 
estimate that students incidentally in close proximity are friends. To exclude the contingency, we should consider the appropriate time (i.e., how many seconds inside the viewable area) for students to be accurately judged to have common interests. In other words, we should consider a condition to update the friendship matrix.

Existing friendship: If DSID and LMID are the same among students in a similar period, the students can be considered existing friends with a common interest in the same learning material because friends often enter the viewable area together. Whether the viewing period is similar is determined according to the following criteria.

- The difference in the start viewing time of the same learning material is within $1 \mathrm{~s}$.

- The difference in the end viewing time of the same learning material is within $1 \mathrm{~s}$.

In Table 1 and Fig. 5, two students (SID = 1 and 2) and three students (SID = 2, 4, and 5) are estimated to be existing friends with common interest in the same learning material (LMID = 1). This estimation does not consider friend-of-friend as existing friends. We assume that once students become existing friends, the friendship continues indefinitely.

Potential friendship: If LMID is the same but the viewing time or place (i.e., DSID) differs among students, a group of students can be considered potential friends with common interests. In this estimation, the criteria of similarity of viewing period is identical with that of the existing friendship. Thus, students who viewed a similar part of a given learning material at a different time or place are estimated to have potential friendship. For example, in Table 1 and Fig. 5, two students (SID = 1 and 6) and three students ( $\mathrm{SID}=4,9$, and 10) have viewed the same learning material (LMID = 1) using different NLSs (DSID = 1 and 2). Based on a common interest, these students can become friends in the future.

Table 1. Viewing data

\begin{tabular}{|c|c|c|c|c|c|c|}
\hline SID & DSID & LMID & STD $^{*}$ & ETD $^{*}$ & STV $^{*}$ & ETV $^{*}$ \\
\hline 1 & 1 & 1 & $10: 15.00$ & $10: 20.00$ & $10: 15.00$ & $10: 16.15$ \\
\hline 2 & 1 & 1 & $10: 15.00$ & $10: 20.00$ & $10: 15.01$ & $10: 16.16$ \\
\hline 3 & 1 & 1 & $10: 15.00$ & $10: 20.00$ & 10.15 .01 & $10: 16.18$ \\
\hline 4 & 1 & 1 & $10: 15.00$ & $10: 20.00$ & $10: 15.02$ & $10: 16.16$ \\
\hline 5 & 1 & 1 & $10: 15.00$ & $10: 20.00$ & $10: 15.02$ & $10: 16.15$ \\
\hline 6 & 2 & 1 & $10: 15.00$ & $10: 20.00$ & 10.15 .00 & $10: 16.14$ \\
\hline 7 & 2 & 1 & $10: 15.00$ & $10: 20.00$ & $10: 15.00$ & $10: 15.03$ \\
\hline 8 & 2 & 1 & $10: 15.00$ & $10: 20.00$ & $10: 15.02$ & $10: 16.13$ \\
\hline 9 & 2 & 1 & $10: 15.00$ & $10: 20.00$ & 10.15 .03 & $10: 16.15$ \\
\hline 10 & 2 & 1 & $10: 15.00$ & $10: 20.00$ & $10: 15.03$ & $10: 16.17$ \\
\hline
\end{tabular}

STD: Start time of delivering LM

ETD: End time of delivering LM

STV: Start time of viewing LM

ETV: End time of viewing LM

* Date is omitted. 
Table 2. Friendship matrix

\begin{tabular}{|c|c|c|c|c|c|c|c|c|c|c|}
\hline SID & $\mathbf{1}$ & $\mathbf{2}$ & $\mathbf{3}$ & $\mathbf{4}$ & $\mathbf{5}$ & $\mathbf{6}$ & $\mathbf{7}$ & $\mathbf{8}$ & $\mathbf{9}$ & $\mathbf{1 0}$ \\
\hline 1 & - & $\mathrm{E}$ & $\mathrm{N}$ & $\mathrm{N}$ & $\mathrm{N}$ & $\mathrm{P}$ & $\mathrm{N}$ & $\mathrm{N}$ & $\mathrm{N}$ & $\mathrm{N}$ \\
\hline 2 & $\mathrm{E}$ & - & $\mathrm{N}$ & $\mathrm{E}$ & $\mathrm{E}$ & $\mathrm{N}$ & $\mathrm{N}$ & $\mathrm{N}$ & $\mathrm{N}$ & $\mathrm{N}$ \\
\hline 3 & $\mathrm{~N}$ & $\mathrm{~N}$ & - & $\mathrm{N}$ & $\mathrm{N}$ & $\mathrm{N}$ & $\mathrm{N}$ & $\mathrm{N}$ & $\mathrm{N}$ & $\mathrm{N}$ \\
\hline 4 & $\mathrm{~N}$ & $\mathrm{E}$ & $\mathrm{N}$ & - & $\mathrm{E}$ & $\mathrm{N}$ & $\mathrm{N}$ & $\mathrm{N}$ & $\mathrm{P}$ & $\mathrm{P}$ \\
\hline 5 & $\mathrm{~N}$ & $\mathrm{E}$ & $\mathrm{N}$ & $\mathrm{E}$ & - & $\mathrm{N}$ & $\mathrm{N}$ & $\mathrm{N}$ & $\mathrm{N}$ & $\mathrm{N}$ \\
\hline 6 & $\mathrm{P}$ & $\mathrm{N}$ & $\mathrm{N}$ & $\mathrm{N}$ & $\mathrm{N}$ & - & $\mathrm{N}$ & $\mathrm{N}$ & $\mathrm{N}$ & $\mathrm{N}$ \\
\hline 7 & $\mathrm{~N}$ & $\mathrm{~N}$ & $\mathrm{~N}$ & $\mathrm{~N}$ & $\mathrm{~N}$ & $\mathrm{~N}$ & - & $\mathrm{N}$ & $\mathrm{N}$ & $\mathrm{N}$ \\
\hline 8 & $\mathrm{~N}$ & $\mathrm{~N}$ & $\mathrm{~N}$ & $\mathrm{~N}$ & $\mathrm{~N}$ & $\mathrm{~N}$ & $\mathrm{~N}$ & - & $\mathrm{N}$ & $\mathrm{N}$ \\
\hline 9 & $\mathrm{~N}$ & $\mathrm{~N}$ & $\mathrm{~N}$ & $\mathrm{P}$ & $\mathrm{N}$ & $\mathrm{N}$ & $\mathrm{N}$ & $\mathrm{N}$ & - & $\mathrm{P}$ \\
\hline 10 & $\mathrm{~N}$ & $\mathrm{~N}$ & $\mathrm{~N}$ & $\mathrm{P}$ & $\mathrm{N}$ & $\mathrm{N}$ & $\mathrm{N}$ & $\mathrm{N}$ & $\mathrm{P}$ & - \\
\hline
\end{tabular}

E: Existing friendship

P: Potential friendship

N: No friendship

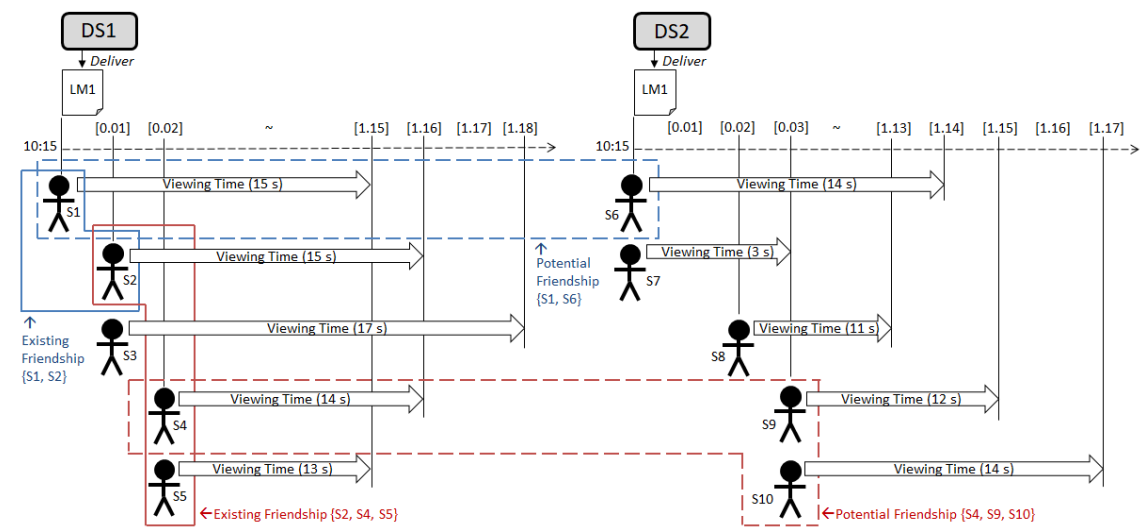

Fig. 5. Friendship estimation

\subsection{Delivery mechanism}

The extended NLS delivers learning materials based on the friendship matrix. There are two delivery modes corresponding to the two types of friendship, i.e., existing and potential. The two delivery modes are switched according to settings described in the delivery schedule.

Delivery based on existing friendship: The extended NLS (i.e., the client system) identifies a student entering the viewable area and immediately begins the following procedure for delivery based on existing friendships.

1. The client system identifies students who enter the viewable area within $1 \mathrm{~s}$.

2. The client system sends the students' IDs to the server system.

3. The server system receives the IDs and retrieves their existing friends from the friendship matrix ("E" in Table 2).

4. The system finds the largest group of existing friends. 
5. The server system selects the most common learning material among the existing friends that has not been viewed from beginning to end.

6. The server system sends the selected LMID to the client system.

7. The client system receives the LMID and presents the corresponding learning material from the beginning in an interruption manner.

In Fig. 4, when S1 first enters the viewable area, followed immediately by three students, LM1 is delivered because S2, S4, and S5 are in the largest group of existing friends, and these three students have not viewed LM1 fully. This delivery method will provide existing friends with an opportunity to learn together (e.g., discuss a topic in the delivered material) and reinforce their existing friendship.

Delivery based on potential friendship: Similarly, the extended NLS begins with the following procedure for delivery based on potential friendship.

1. The client system identifies students who enter the viewable area within $1 \mathrm{~s}$.

2. The client system sends the student ID and identifies students' IDs to the server system.

3. The server system receives the IDs and retrieves the students' potential friends from the friendship matrix ("P" in Table 2).

4. The system finds the largest group of potential friends.

5. The server system selects the most common learning material among the potential friends that has not been viewed from beginning to end.

6. The server system sends the selected LMID to the client system.

7. The client system receives the LMID and presents the corresponding learning material from the beginning in an interruption manner.

In Fig. 4, when S6 first enters the viewable area, followed immediately by three other students, LM1 is delivered because S9 and S10 are potential friends who have not viewed LM1 fully. This delivery method will help potential friends recognize each other and will provide an opportunity to learn and discuss, thereby providing opportunities to develop potential friendships into a learning community (i.e., existing friendship).

\section{$4 \quad$ Preliminary Experiment}

The accuracy of friendship estimation should be examined prior to the effectiveness of the NLS because the delivery mechanism for satisfying LC3 and LC4 assumes that the friendship estimation is accurate. Although the definition of friendship varies by individual, every student can enumerate their existing friends. However, students cannot enumerate potential friends, i.e., who will become friends. Thus, we conducted a preliminary experiment to examine the accuracy of existing-friendship estimation. 


\subsection{Focus}

The existing-friendship estimation includes several undetermined elements. For example, if students are inside the viewable area for $1 \mathrm{~s}$ simultaneously, they are estimated to be existing friends. We had not previously examined whether a single short period of simultaneous presence in the viewable area was appropriate. Students who are inside the viewable area simultaneously for a longer time may have stronger existing friendships. In addition, the more frequently students meet inside the viewable area, the stronger their existing friendships may become. To exclude the contingency and increase the accuracy, we should add an element concerning viewing frequency and set appropriate values.

In the experiment, we focused on two methods of existing-friendship estimation, i.e., viewing-time-based and viewing-frequency-based methods, and attempted to clarify the appropriate values.

\subsection{Settings}

For safety reasons, a few restrictions were imposed on the experiment. To avoid congestion caused by static students, we had to install a single NLS alternately in two elevator halls with lounge areas on the same floor in a main lecture building of Tokushima University only around the time of a lecture. In addition, to avoid directly disturbing students' concentration in the lounge areas, we had to install the NLS away from lounge seats or turn the display toward the hallway. The RFID reader was positioned immediately under the display, facing front. A diagram and photographs of the installation locations (eastern and western elevator halls) are shown in Figure 6.

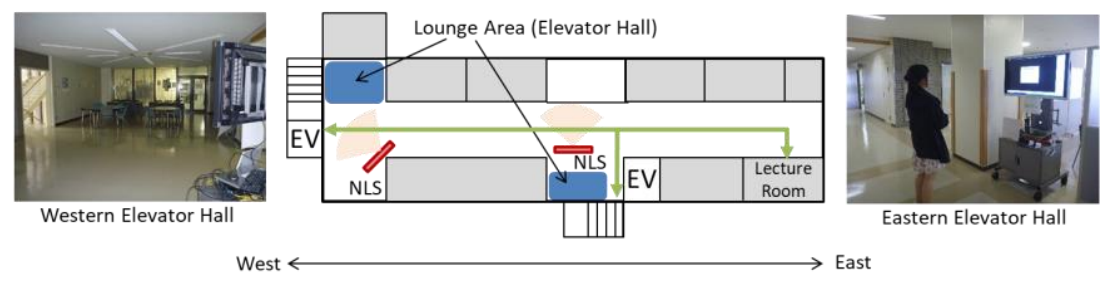

Fig. 6. NLS installed in elevator halls

Participants: We asked first-year undergraduate students who attended a lecture on "discrete mathematics" in a computer science course to participate in the experiment. We explained that we needed to ask them to secretly inform us of their existing friendships. We promised that we would consider such information as private and would never disclose their friendships. In the end, 84 students participated in the experiment. Each participant was given an RFID tag, and the participants were asked to bring the tag when attending the lecture.

NLS: We installed the NLS in the eastern and western elevator hallways before and after the lecture, respectively. The NLS delivered five-minute-long videos about four popular topics (e.g., English conversation and disaster management) evenly in terms of 
delivery frequency and simultaneously collected viewing data for 40 minutes before and after the lecture every week for approximately three months.

Procedure: Figure 7 outlines the experimental procedure. At the first lecture, we gave an RFID tag to each participant and recorded the RFID tag-participant relation (i.e., ID and participant name). The viewing data were collected from the second to the thirteenth lecture. After the fourteenth lecture, we collected participants' existing friendships via an online survey using a web-based questionnaire system. The questionnaire asked participants to "Please write the full names of your friends in the same course. You can determine the definition of friends."

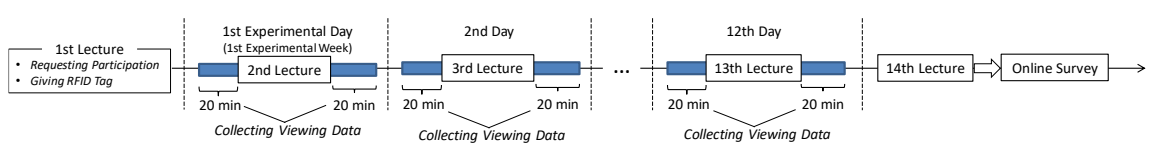

Fig. 7. Experimental procedure

\subsection{Results}

Figure 8 illustrates the existing friendships collected via the online survey, i.e., real friendships $\left(F_{\text {real }}\right)$, as an undirected graph where unidirectional friendships were allowed. There were 391 real existing friendships (links) among 83 participants (nodes). Note, one participant did not complete the online survey.

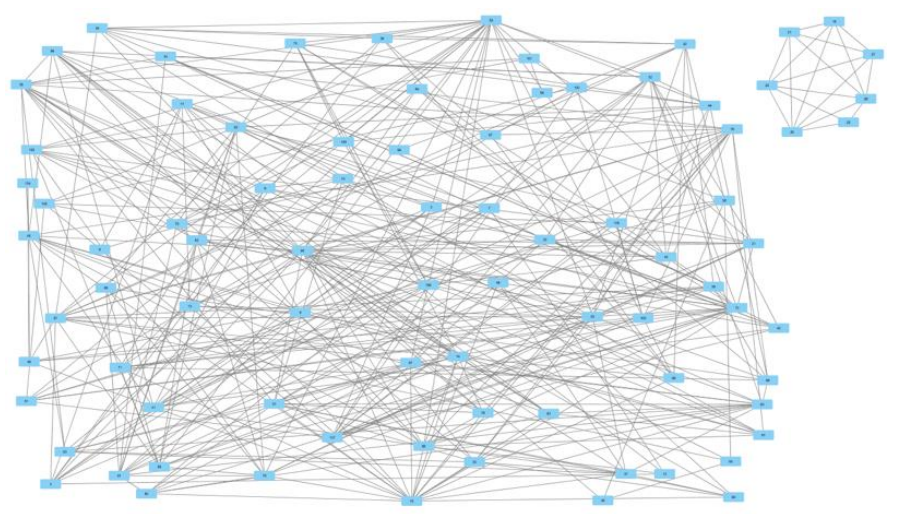

Fig. 8. Real existing friendships collected via online survey

On average, on each day of the experiment, approximately 20 participants were identified. However, we observed that more participants passed in front of the display than were identified. The NLS could not identify participants accurately due to participantcaused physical interference in reading RFID tags. We think that the NLS cannot estimate existing friendships accurately in a single short period; although, identification accuracy may also depend on the location of the NLS. In other words, the NLS should estimate existing friendships from the viewing data accumulated for a certain period of time. Thus, we accumulated viewing data for 12 days for approximately three months 
and then estimated existing friendships $\left(F_{\text {estimated }}\right)$ among participants from the accumulated data. Tables 3 and 4 show the results regarding estimated existing friendships by viewing time (1-5 s) and viewing frequency (2-5 times), respectively. The NLS identified 69 participants; 15 participants were never identified.

Viewing time: In Table 3, the case of viewing time $(V T=1)$ and number of participants $(n(P)=69)$ means that 69 participants were inside the viewable area for $1 \mathrm{~s}$ or more over the duration of the experiment (12 days). Most participants were inside the viewable area for less than $4 \mathrm{~s}$. We observed that most participants did not stop to view the learning materials. These results indicate that they needed approximately $3 \mathrm{~s}$ to leave the viewable area by walking. In our analysis, Recall values ranged from $0.008(V T=$ 5) to $0.281(V T=1)$. The Precision values ranged from $0.46(V T=1)$ to $1.00(V T=5)$. The estimated existing friendships were identical to the real ones when $V T=5$. As the time the participants were in the viewable area increased, the Recall and Precision values decreased and increased, respectively, which indicates a trade-off relationship between Recall and Precision. The F-measure values ranged from $0.015(V T=5)$ to 0.349 $(V T=1)$.

$$
\begin{gathered}
\text { Recall }=\frac{n\left(F_{\text {real }} \cap F_{\text {estimated }}\right)}{n\left(F_{\text {real }}\right)} \\
\text { Precision }=\frac{n\left(F_{\text {real }} \cap F_{\text {estimated }}\right)}{n\left(F_{\text {estimated }}\right)} \\
F=\frac{2 \times \text { Recall } \times \text { Precision }}{\text { Recall }+ \text { Precision }}
\end{gathered}
$$

Table 3. Viewing data results

\begin{tabular}{|c|c|c|c|c|c|c|}
\hline VT & $\boldsymbol{n}(\boldsymbol{P})$ & $\boldsymbol{n}\left(\boldsymbol{F}_{\text {estimated }}\right)$ & $\boldsymbol{n}\left(\boldsymbol{F}_{\text {correct }}\right)$ & Recall & Precision & $\boldsymbol{F}$ \\
\hline 1 & 69 & 239 & 110 & 0.281 & 0.460 & 0.349 \\
\hline 2 & 67 & 155 & 86 & 0.220 & 0.555 & 0.315 \\
\hline 3 & 54 & 64 & 41 & 0.105 & 0.641 & 0.180 \\
\hline 4 & 26 & 21 & 15 & 0.038 & 0.714 & 0.073 \\
\hline 5 & 5 & 3 & 3 & 0.008 & 1.00 & 0.015 \\
\hline
\end{tabular}

VT: Lower limit of viewing time (s)

$n(P)$ : Total number of different participants identified by the RFID reader $n\left(F_{\text {estimated }}\right)$ : Number of estimated existing friendships

$n\left(F_{\text {correct }}\right)$ : Number of correctly estimated existing friendships, i.e., $n\left(F_{\text {real }} \cap F_{\text {estimated }}\right)$

Table 4. Viewing frequency results

\begin{tabular}{|c|c|c|c|c|c|c|}
\hline VF & $\boldsymbol{n}(\boldsymbol{P})$ & $\boldsymbol{n}\left(\boldsymbol{F}_{\text {estimated }}\right)$ & $\boldsymbol{n}\left(\boldsymbol{F}_{\text {correct }}\right)$ & Recall & Precision & $\boldsymbol{F}$ \\
\hline 1 & 69 & 239 & 110 & 0.281 & 0.460 & 0.349 \\
\hline 2 & 45 & 57 & 44 & 0.113 & 0.772 & 0.196 \\
\hline 3 & 27 & 24 & 20 & 0.051 & 0.833 & 0.196 \\
\hline 4 & 22 & 17 & 14 & 0.036 & 0.824 & 0.069 \\
\hline 5 & 20 & 13 & 10 & 0.026 & 0.769 & 0.050 \\
\hline
\end{tabular}

VF: Viewing frequency (s) 
Viewing frequency: In Table 4, the case of $V F=2$ and $n(P)=45$ means that 45 participants (approximately two thirds of 69 participants) were inside the viewable area for $2 \mathrm{~s}$ or more simultaneously on two different days. $V T=1$ was constant in all VFs. Twenty participants were inside the viewable area simultaneously on five different days. Here, the Recall values ranged from $0.026(V F=5)$ to $0.281(V F=1)$. The Precision values ranged from $0.460(V F=1)$ to $0.833(V F=3)$. The Recall value decreased the more frequently participants were in the viewable area. The Precision values did not change significantly except for $V F=1$; the Precision values remain high after $V F$ $=2$. The $F$-measure values ranged from $0.050(V F=5)$ to $0.349(V F=1)$.

\subsection{Considerations}

Regarding both viewing time and frequency, the calculated Recall values were not favorable, and the calculated Precision values were relatively favorable. The $F$-measure values, which were reduced due to low Recall values, were not favorable relative to comprehensive accuracy. These results indicate that the estimated existing friendships could be more than $70 \%$ correct when $V T \geq 4$ and $V F \geq 2$; however, the estimated existing friendships did not fully cover the real existing friendships. Thus, we think that delivery based on existing friendship cannot correctly find the largest group of existing friends that are in front of the display. LC3, which requires high Recall and Precision values for the existing-friendship estimation, can result in low effectiveness.

In terms of $F$-measure, there is no remarkable difference between viewing-timebased and viewing-frequency-based methods. The viewing-time-based method is expected to be useful when the estimation needs to be done with fewer displays (and more learning materials), i.e., students have fewer opportunities to view a learning material simultaneously. The viewing-frequency-based method, which can maintain relatively high Precision values even with lower viewing frequency, is expected to be useful when more displays are installed. In any case, for higher estimation accuracy, we need to significantly improve Recall. In addition to installing the NLS in more locations, one possible approach to improving the system is to identify students more robustly. For example, by installing the RFID reader on the ceiling pointing in a downward direction, we may be able to reduce the physical interference that caused problems reading RFID tags.

In the experiment, existing-friendship estimation was inaccurate; however, that could change under different experimental settings, i.e., the NLS installation location and the periods of learning material delivery. Our experimental results indicated that most participants did not stop to view the learning materials, and this unexpected situation has not changed since the NLS was initially installed in public spaces on the university campus. If participants were given a proper space and sufficient time to stop to view the learning material, they could stay in the viewable area longer. Thus, the inaccurate estimation and associated low Recall values could be improved due to longer viewing times. As a result, in the experiment, we examined whether the existing-friendship estimation was applicable to passersby in front of the display. We did not originally envision such applicability. 
From the above, it is evident that the existing-friendship estimation is currently inaccurate and unsuitable for LC3. However, we believe that we can improve the existingfriend estimation by installing the NLSs and the RFID reader in more appropriate locations and only using the viewing data for students who stop to view learning materials.

\section{Conclusion}

This paper has described an innovative DS system that delivers learning materials based on student friendships so that students can continually learn together. To realize the DS system, we have extended the NLS based on LC concepts to reinforce existing friendship (LC3) and identify potential friendship (LC4). These friendships are estimated from the viewing data that are collected by the RFID reader and that represent students' interests for the given learning material, i.e., when (how long), where (which viewable area), and what (which learning materials the students viewed). We conducted a preliminary experiment at a university campus to examine the accuracy of an existingfriendship estimation method. The experiment revealed that the estimation did not work as intended and the accuracy was not high. If the accuracy is not guaranteed, the extended NLS cannot deliver learning materials that satisfy the LCs. To improve the accuracy, we should conduct a larger-scale experiment, collect and analyze more viewing data. In addition, we may have to explore other approaches to improving the accuracy. A possible approach is to adopt a real-time vision system for face and gaze recognition using a camera attached to the display.

We have not yet evaluated the potential-friendship estimation, which is almost identical to the existing-friendship estimation. When multiple NLSs are installed in appropriate places, students in different places or at different times will become more frequently aware of the systems and will view their interested learning materials. As a result, potential friendships will be estimated frequently. As we establish suitable settings for the two estimation methods, we need to determine a way to examine whether the potential-friendship estimation is accurate.

Smartphones are popular, convenient personal tools to view rich digital content. Students nowadays may focus on smartphone displays and take no notice of public displays. However, we would like to believe that students can make friends with common interests (i.e., create a learning community) through the NLS, i.e., reinforcing existing friendships and identifying potential friendships in the real-world environments can lead to more effective learning. Mäkelä et al. [16] identified weather, events, surroundings, space, inhabitants, and vandalism as factors that affect public display deployment. Considering these factors, we intend to re-extend the NLS to encourage students to direct their eyes to public displays rather than smartphone displays.

\section{Acknowledgement}

This study was supported in part by a Grant-in-Aid for Young Scientists (A) No. 24680079 from the Japan Society for the Promotion of Science. We thank H. Kangawa for his effort in this study. 


\section{$7 \quad$ References}

[1] Taniguchi, Y. (2018). Content scheduling and adaptation for networked and context-aware digital signage: A literature survey. ITE Transactions on Media Technology and Applications, 6 (1): 18-29. https://doi.org/10.3169/mta.6.18

[2] Müller, J, Eberle, D. and Tollmar, K. (2014). Communiplay: A field study of a public display mediaspace. Proceedings of the SIGCHI Conference on Human Factors in Computing Systems (CHI'14): 1415-1424. https://doi.org/10.1145/2556288.2557001

[3] Häkkilä, J., Koskenranta, O., Posti, M., Ventä-Olkkonen, L. and Colley A. (2013). Clearing the virtual window: Connecting two locations with interactive public displays. Proceedings of the 2nd ACM International Symposium on Pervasive Displays (PerDis'13): 85-90. https://doi.org/10.1145/2491568.2491587

[4] Zhang, Y., Müuller, J., Chong, M., Bulling, A. and Gellersen, H. (2014). GazeHorizon: Enabling passers-by to interact with public displays by gaze, Proceedings of the 2014 ACM International Joint Conference on Pervasive and Ubiquitous Computing (UbiComp'14): 559563. https://doi.org/10.1145/2632048.2636071

[5] Ojala, T., Kostakos, V., Kukka, H., Heikkinen, T., Linden, T., Jurmu, M., Hosio, S., Kruger, T. and Zanni, D. (2012). Multipurpose interactive public displays in the wild: Three years later. IEEE Computer, 45 (5): 42-49. https://doi.org/10.1109/mc.2012.115

[6] Ng, P.C., She, J., Jeon, K.E. and Baldauf, M. (2017). When smart devices interact with pervasive screens: A survey. ACM Trans. Multimedia Comput. Commun. 13 (4): Article 55. https://doi.org/10.1145/3115933

[7] Hossain, M.A., Islam, A., Le, N.T., Lee, Y.T., Lee, H.W. and Jang, Y.M. (2016). Performance analysis of smart digital signage system based on software-defined IoT and invisible image sensor communication. International Journal of Distributed Sensor Networks, 12(7): 1-14. https://doi.org/10.1177/1550147716657926

[8] Kim. S., Park, J., Chae, M., Jung, S. and Chang H. (2019). A device binding method based on content illumination pattern in public display environments. PLoS ONE 14(5): e0214493, https://doi.org/10.1371/journal.pone.0214493

[9] Brignull, H., Izadi, S., Fitzpatrick, G., Rogers, Y., and Rodden, T. (2004). The introduction of a shared interactive surface into a communal space. Proc. of the 2004 ACM Conference on Computer Supported Cooperative Work (CSCW'04): 49-58. https://doi.org/10.1145 $\underline{1031607.1031616}$

[10] Sorce, S., Gentile, V., Enea, C., Gentile, A., Malizia, A., Milazzo, A. (2017). A touchless gestural system for extended information access within a campus. Proceedings of the 2017 ACM Annual Conference on SIGUCCS: 37-43. https://doi.org/10.1145/3123458. 3123459

[11] Kang, J., Lindgren, R. and Planey, J. (2018). Exploring emergent features of student interaction within an embodied science learning simulation. Multimodal Technologies and Interaction, 2 (39): https://doi.org/10.3390/mti2030039

[12] Matsumoto, J., Mitsuhara, H., Uosaki, N., Teshigawara, M. and Yano, Y. (2009). Learning Material Creation by Student Participation for Niche-Learning. IADIS International Journal on WWW/Internet 7(2): 31-46

[13] Mitsuhara, H. (2012). An Interactive Digital Signage System Using Live-video Superimposing and Quiz Slideshow. The Journal of Information and Systems in Education 11(1): 6268. https://doi.org/10.12937/ejsise.11.62

[14] Mitsuhara, H. and Shishibori, M. (2014). Digital Signage System for Learning Material Presentation Based on Learning Continuum. Proc. of International Conference on Smart 
Learning Environments (ICSLE'14): 121-128. https://doi.org/10.1007/978-3-662-44188$\underline{6 \quad 19}$

[15] Hardy, J., Rukzio, E. and Davies, N. (2011). Real world responses to interactive gesture based public displays. Proceedings of the 10th International Conference on Mobile and Ubiquitous Multimedia (MUM'11): 33-39. https://doi.org/10.1145/2107596.2107600

[16] Mäkelä, V., Sharma, S., Hakulinen, J., Heimonen, T. and Turunen, M. (2018). Challenges in public display deployments: a taxonomy of external factors. Proceedings of the $2017 \mathrm{CHI}$ Conference on Human Factors in Computing Systems (CHI'17): 3426-3475. https://doi.org/10.1145/3025453.3025798

\section{Authors}

Hiroyuki Mitsuhara received the B.E. and M.E. degrees from Kindai University in 1998 and 2000, and then he received the Ph.D. degree from Tokushima University in 2003. He is currently an associate professor at Tokushima University. His interest includes interactive learning systems, game-based learning, VR/AR, and disaster education. E-mail: mituhara@is.tokushima-u.ac.jp

Masami Shishibori received his BS Degree in 1991, his MS Degree in 1993 and PhD Degree in 1997, from Tokushima University, Japan. He is currently a full professor at Tokushima University, Japan. His research interests include multimedia processing, information retrieval, and natural language processing.

Article submitted 2020-03-10. Resubmitted 2020-04-06. Final acceptance 2020-04-07. Final version published as submitted by the authors. 